\title{
Vibrissal anesthesia and suppression of irritable fighting in rats: A temporary duration of effect in experienced fighters*
}

\author{
DONALD H. THOR and WILLIAM B. GHISELLI \\ Edward R. Johnstone Training and Research Center, Bordentown, New Jersey 08505
}

\begin{abstract}
Selected pairs of agressive rats fighting reliably and at relatively high levels were successively treated with lidocaine anesthesia of the vibrissal pads prior to each daily test session. The initial decrement in fighting was substantial, but after only three sessions pretest level of fighting was regained. Initial decrease in fighting of a second group following removal of all vibrissae was comparable to that produced by local anesthesia, but recovery of fighting to pretest levels was slower and less complete. The results suggest that intact and functional vibrissal organs are not mandatory for normal fighting when Ss are given sufficient preliminary fighting experience to footshock. Applications of vibrissal sensory deprivation techniques for control of fighting in experimental designs calling for repeated measures or sequential treatments are potentially liable to confounding.
\end{abstract}

Historically, investigators have long recognized the import of rodent vibrissal organs in providing proximal sensory information. Early monographs by Richardson (1909) and Vincent (1912) reveal interests stemming from reports of successful maze performance by blind and anosmic rats and the presumed interactive roles of the senses as defined by characteristics of the experimental task. Watson (1907) compared the maze performance of blind, anosmic, and normal rats before and after being devibrissaed. Although immediate performance was impaired, no decrement in running time was observed $24 \mathrm{~h}$ after being devibrissaed; Watson concluded that removal of the vibrissae had no effect when sufficient time was allowed for adaptation. Richardson (1909) also found that tactual sensation from the vibrissae was unnecessary after learning had occurred in a platform jumping task.

More recently, Schiffman, Lore, Passafiume, and Neeb (1970) tested hooded and albino rats on a visual cliff apparatus providing both visual and tactual depth cues. Devibrissaed rats showed a marked preference for descent on the shallow side of the visual cliff, whereas intact controls showed no significant preference for either side. Rats apparently do not apply visual information unless forced to do so by a center-board height preventing vibrissal contact with the glass surfaces. Tactual information from the vibrissae may thus be prepotent over visual information on this depth-perception test. Schiffman et al (1970) suggested that visual discrimination on a number of visual tasks may be substantially improved by removal of the vibrissae.

Clipping of the vibrissae (Bugbee \& Eichelman, 1972)

*This research was supported in part by NIH Research Grant MH 21577-02. Reprint requests may be addressed to either author. or obstructing their free movement (Flory, Ulrich, \& Wolff, 1965) decreased fighting of paired rats exposed to momentary footshock. Anesthesia of the vibrissal pads also decreased aggressive responding to footshock (Thor \& Ghiselli, 1973a, b) or to centrally induced fighting following intraperitoneal injection of apomorphine hydrochloride (Thor \& Ghiselli, in press).

The noted capacity of rodents to overcome deprivation of vibrissal sensation with task experience (as originally reported by Richardson, 1909; Vincent, 1912; and Watson, 1907) may also apply to aggressive behavior. Hence, the present experiment was conducted to test the permanence of suppression in fighting by facial anesthesia with pairs of rats known to exhibit high and stable levels of aggressive responding to footshock. Devibrissaed pairs were also tested to determine any comparable effects attributable to absence of all vibrissae.

\section{METHOD}

\section{Subjects}

Twenty-four 150-day-old male Long-Evans rats were selected from a larger pool reared from breeders originally purchased from a commercial source (Rockland, Gilbertsville, Pennsylvania). They were housed four animals per pan cage, and received ad lib food and water. Each animal had received 14 preliminary daily shock-fight exposures with noncagemates in the test apparatus and was selected on the basis of reliable fighting response to footshock.

\section{Apparatus}

The fight enclosure was a standard Lafayette rodent operant chamber withou ${ }^{\dagger}$ manipulanda. Standard electromechanical programming delivered neon-scrambled shock from a constant-current shock source to the stainless steel grid floor. The chamber was contained within an acoustic enclosure and was diffusely illuminated by an overhead incandescent lamp (1300 lux at cage floor). 


\section{Procedure}

Twelve pairs of noncagemates were formed on the basis of comparable weight and were tested on three daily sessions to determine baseline level of shock-elicited fighting. Two matched groups of six pairs each were then formed on the basis of the pretest fighting scores. As in the preliminary screening, each daily session consisted of 50 shocks of $0.5 \mathrm{sec}$ duration at $2.0 \mathrm{~mA}$ intensity, presented at 3.0 -sec intervals.

All subjects in the devibrissaed group were shaved of all vibrissae (with electric clippers) $24 \mathrm{~h}$ prior to initial experimental test and at 3-day intervals thereafter to prevent significant regrowth. All subjects in the anesthesia group were etherized and given $0.2 \mathrm{ml}$ of $1 \%$ lidocaine hydrochloride with epinephrine $1: 100,000$ subcutaneously in the caudal portion of each vibrissal pad $30 \mathrm{~min}$ prior to each test session. The anesthetic rendered subjects unresponsive to needle pricks of the pads and effected a paralysis of vibrissal movement. Both groups were tested consecu tively at the same time each day in alternating order. The test chamber was thoroughly cleansed after each group finished a session, and the test enclosure was fan-ventilated throughout the testing period.

Three experienced observers scored fighting after the general criteria described by Eichelman (1972), which define an attack as consisting of directed movement by at least one member toward the other, resulting in physical contact and followed by at least one of four behaviors: biting, boxing, mutual upright threat posturing, or supine submissive posturing. Fighting was expressed as the percent of shocks effective in eliciting attack for each pair.

\section{RESULTS}

Means and standard errors of fight scores for each group on each session are presented in Fig. 1. Both groups fought at identically high levels during pretest sessions (73\%) and were comparable in original depression of fighting following removal of vibrissae or application of lidocaine anesthesia to the vibrissal pads. The decrease in fighting was significant for both anesthesia $(t=4.99, \mathrm{df}=5, \mathrm{p}<.01)$ and devibrissaed $(t=6.26, d f=5, p<.002)$ groups. Mean fight score of the anesthesia group was also significantly depressed on Session $5(\mathrm{t}=3.84, \quad \mathrm{df}=5, \quad \mathrm{p}<.02)$ and was nonsignificant thereafter. Session mean fighting scores of the devibrissaed group remained significantly lower $(\mathrm{p}<.05)$ than pretest through Session 7 , but did not differ significantly (ps $>.05$ ) from pretest level on Sessions 8, 10,12,13, and 14 (all $t$ tests two-tailed). Greater variability of fight scores in the devibrissaed group may be attributed to two pairs of animals which failed to recover pretest fighting capacity, even after 11 test sessions (attack level of $6 \%$ and $16 \%$ on Session 14). Remaining pairs of devibrissaed animals all fought consistently at or above pretest levels. Conversely, all anesthetized pairs recovered to pretest level of aggressive responding.

\section{DISCUSSION}

The results indicate that shaving the vibrissae and local perioral anesthesia are equally effective in initially suppressing shock-elicited fighting of experienced fighting pairs, confirming previous observations

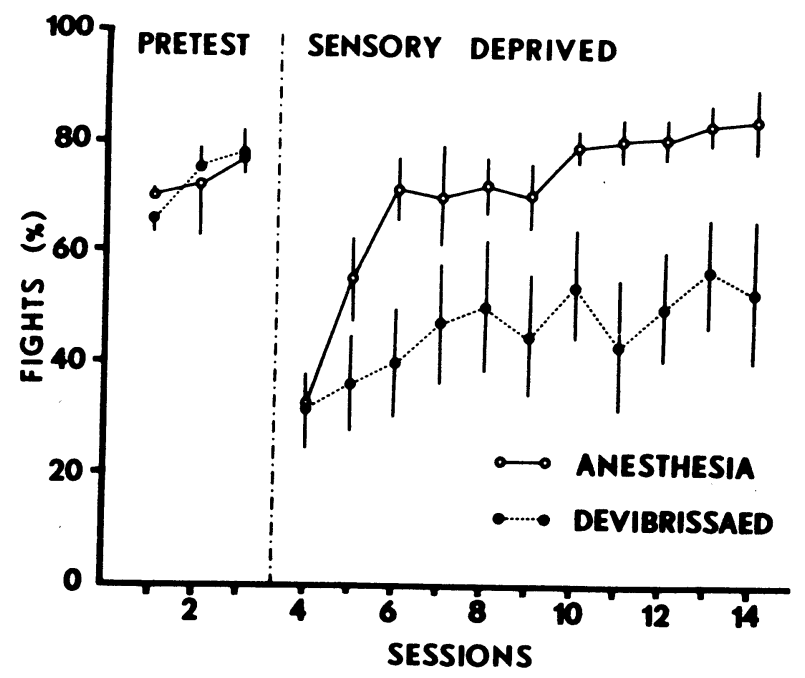

Fig. 1. Means and standard errors (vertical bars) of anesthetized and devibrissaed pairs over consecutive daily sessions.

regarding efficacy of both techniques (Bugbee \& Eichelman, 1972; Thor \& Ghiselli, 1973b). Within three daily sessions, however, anesthesia treated experienced fighters fought at pretest baseline. Although fighting remained at low levels in some devibrissaed pairs, the majority regained or exceeded pretest baseline. Evidently, other sensory modalities are capable of orienting and guiding normal and effective attack behavior when deprived of vibrissal or perioral dermal sensation.

Variability of fighting in the devibrissaed group was greater than that of the anesthetized group. One explanation may involve a greater sensory disorientation in devibrissaed animals due to a continuous lack of sensory input from the vibrissae; in comparison, animals injected with the anesthetic were only deprived of facial sensation for a few hours each day (Thor \& Ghiselli, 1973b). Another explanation may hinge on the lack of sensitivity to pain conjointly present with loss of vibrissal input in the anesthetized condition. Anesthetized subjects, when occasionally bitten on the snout, failed to emit "cut-off" behaviors characteristic of retreat (Chance, 1962) which may have elevated level of fighting. Cut-off behaviors were observed in devibrissaed subjects and, coupled with the potential punishment of bite-induced pain, could account for their lower level of fighting. The anesthetized group sustained more trauma from bites and scratches that may have contributed to greater overall discomfort, thus indirectly increasing level of fighting. While the source of the difference between the effects of devibrissaing and anesthesia of the vibrissal pad remains to be specified, it appears to be sensory in nature. Previous informal observations have shown that rats which receive subcutaneous lidocaine injections in the middorsal 
epidermis fight normally, indicating that the anesthetic is not acting systemically. Rats with buccal and mandibular branches of the facial nerve bilaterally sectioned also fight, indicating that the paralysis of the vibrissae produced by lidocaine is not a key factor. Rats trained to find buried cheese continue to locate the cheese under vibrissal anesthesia, demonstrating a lack of olfactory involvement when lidocaine is injected into the vibrissal pads.

In general, the nature of the attack behavior observed in the majority of devibrissaed or anesthetized pairs was comparable to that of untreated pairs. No major difficulty in orientation or localization in reference to an opponent was apparent after several sessions of exposure. It may be emphasized, however, that two pairs of devibrissaed animals remained at unusually low fight levels after numerous postdeprivation sessions. The latter observation supports the position that removal of vibrissae and anesthesia of the vibrissal pad may differ in some behavioral consequences. Loss of the vibrissae results in a temporary depression of shock-elicited attack in either naive or experienced rats; in contrast, local anesthesia of the vibrissal pad results in a total absence of fighting in naive rats and only a temporary suppression of fighting in experienced rats (Thor, Ghiselli, \& Lambelet, in press).

In view of the apparent temporary effect of vibrissal anesthesia in suppressing irritable fighting of experienced rats, the general method as originally proposed (Thor \& Ghiselli, 1973a, b) has a serious limitation with inherent potential for confounding in experimental designs requiring repeated measures or sequential treatments. Detailed examination of behavior during initial exposure to footshock in the experimental context (including the presence of another rat) may suggest a more definitive analysis of the presumed interaction of prior experience with imposed deprivation of cutaneous sensation from the snout.

\section{REFERENCES}

Bugbee, N. M., \& Eichelman, B. S., Jr. Sensory alterations and aggressive behavior in the rat. Physiology \& Behavior, 1972, 8, $\mathbf{9 8 1 - 9 8 5}$

Chance, M. R. A. An interpretation of some agonistic postures: The role of "cut-off" acts and postures. Zoological Society of London, 1962, Symposium No. 8, 71-89

Eichelman, B. S., Jr. Effects of subcortical lesions on shock-induced aggression in the rat. Journal of Comparative \& Physiological Psychology, 1971, 74, 331-339.

Flory, R. K., Ulrich, R. E., \& Wolff, P. C. The effects of visual impairment on aggressive behavior. The Psychological R ecord, $1965,15,185-190$.

Richardson, F. A study of sensory control in the rat. Psychological Review, Monograph Supplements, 1909, No. 12.

Schiffman, H. R., Lore, R., Passafiume, J., \& Neeb, R. Role of vibrissae for depth perception in the rat (Rattus norvegicus). Animal Behaviour, 1970, 18, 290-292.

Thor, D. H. \& Ghiselli, W. B. Suppression of shock-elicited aggression in rats by facial anesthesia. Proceedings of the 81st Annual Convention, APA, Part 2, 1973a, 8, 1025-1026.

Thor, D. H., \& Ghiselli, W. B. Prolonged suppression of irritable aggression in rats by facial anesthesia. Psychological Reports, $1973 \mathrm{~b}, 33,815-820$.

Thor, D. H., \& Ghiselli, W. B. Suppression of mouse-killing and apomorphine-induced social aggression in rats by local anesthesia of the mystacial vibrissae. Journal of Comparative \& Physiological Psychology, in press.

Thor, D. H., Ghiselli, W. B., \& Lambelet, D. C. Sensory control of shock-elicited fighting in rats. Physiology \& Behavior, in press.

Vincent, S. B. The function of the vibrissae in the behavior of the white rat. Behavior Monographs, 1912, 1, No. 5.

Watson, J. B. Kinaesthetic and organic sensations. Psychological Review, Monograph Supplements, 1907, 8, No. 33.

(R eceived for publication June 18, 1974 ; revision received July 30,1974 .) 